\title{
Análise do Tratamento Precoce do Câncer Infantojuvenil no Brasil
}

doi: https://doi.org/10.32635/2176-9745.RBC.2022v68n1.1637

\author{
Analysis of Early Treatment of Childhood Cancer in Brazil \\ Análisis del Tratamiento Temprano del Cáncer Infantil en Brasil
}

Dayane Ketlyn da Cunha Santos'; José Cleyton de Oliveira Santos²; Yuri Barbosa Araujo ${ }^{3}$; Karoline Alves de Almeida; Glebson Santos Sobral'5; Simone Yuriko Kameo6; Glebson Moura Silva7

\section{RESUMO}

Introduçáo: $\mathrm{O}$ tratamento precoce do câncer infantojuvenil possibilita um prognóstico significativo para o paciente, aumentando a sobrevida e diminuindo os riscos de complicaçóes oncológicas, o que torna imperiosa a sua instituição o mais rápido possível. Objetivo: Analisar os fatores associados à instituição precoce do tratamento oncológico na população pediátrica brasileira. Método: Estudo ecológico, com dados de casos de todos os tipos de câncer diagnosticados no Brasil de 2013 a 2019, na população infantojuvenil (0 a 19 anos), disponibilizados no PAINEL-Oncologia. Resultados: Foram incluídos 39.711 casos, dos quais 29.381 (74\%) realizaram o tratamento oportuno, isto é, 30 dias antes do prazo máximo estipulado pela Lei Federal no ${ }^{\circ}$ 12.732/12. Entre os principais fatores associados ao maior tempo até a chegada à instituição terapêutica, destacam-se: neoplasias malignas, com modalidade terapêutica não cirúrgica, residentes da Região Norte, entre 11 e 19 anos, e neoplasias que acometem os olhos e o Sistema Nervoso Central. Conclusáo: Os achados deste estudo apontam dados essenciais, em consonância com a literatura, para visualização da situação atual do tratamento oncológico na população infantojuvenil no Brasil, ao mesmo tempo que demostram problemáticas que, se solucionadas, podem contribuir significativamente para a queda da morbimortalidade.

Palavras-chave: acesso aos serviços de saúde; neoplasias; tempo para o tratamento; assistência integral à saúde; adolescente.

\section{ABSTRACT}

Introduction: The early treatment of childhood cancer should be implemented as soon as possible because it favors a good prognosis for the patient, increasing survival and reducing the risk of oncologic complications. Objective: Understand the factors associated with the early implementation of cancer treatment in the Brazilian pediatric population. Method: Ecological study, with data on cases of all types of cancer diagnosed in Brazil from 2013 to 2019 in the juvenile population (0 to 19 years old) available on PANEL-Oncology. Results: 39,711 cases were included, of which 29,381 (74\%) underwent timely treatment, that is, 30 days before Federal Law 12.732/12-mandated maximum period. Among the main factors associated with more time until the arrival at the therapeutic institution, the following stand out: malignant neoplasms, with non-surgical therapeutic modality, residents of the North region, between 0 and 19 years, and neoplasms affecting the eyes and the central nervous system. Conclusion: The findings of this study reveal essential data in line with the literature, portraying the current situation of cancer treatment in children and adolescents in Brazil, and issues that if resolved can contribute significantly to reduce the morbimortality.

Key words: health services accessibility; neoplasms; time-to-treatment; comprehensive health care; adolescent.

\section{RESUMEN}

Introducción: El tratamiento temprano del cáncer infantil y adolescente permite un pronóstico significativo para el paciente, lo que hace imperativa su institución lo antes posible. Objetivo: Analizar los factores asociados con la institución precoz del tratamiento del cáncer en la población pediátrica brasileńa. Método: Estudio ecológico, con datos de casos de todos los tipos de cáncer diagnosticados en Brasil de 2013 a 2019 en la población juvenil (0 a 19 años) disponibles en el PANEL-Oncología. Resultados: Se incluyeron 39.711 casos, de los cuales 29.381 (74\%) recibieron tratamiento en forma oportuna, es decir, 30 días antes del plazo máximo estipulado por la Ley Federal no. 12.732/12. Entre los principales factores asociados a más tempo hasta la institución terapéutica, se destacan: neoplasias malignas, con modalidad terapéutica no quirúrgica, residentes de la región norte, entre 11 y 19 ańos, y neoplasias que afectan los ojos y el sistema nervioso central. Conclusión: Los hallazgos de este estudio señalaron datos esenciales, en línea con la literatura, visualizar la situación actual del tratamiento del cáncer en niños y adolescentes en Brasil, señalando problemas que, de ser resueltos, pueden contribuir significativamente a la disminución de la morbimortalidad.

Palabras claves: accesibilidad a los servicios de salud; neoplasias; tiempo de tratamiento; atención integral de salud; adolescente.

${ }^{1-7}$ Universidade Federal de Sergipe. Lagarto (SE), Brasil.

1E-mail: daayketlyn27@gmail.com. Orcid iD: https://orcid.org/0000-0002-9312-4891

${ }^{2}$ E-mail: cleyton-121@hotmail.com. Orcid iD: https://orcid.org/0000-0001-5616-7625

${ }^{3}$ E-mail: yuriyba@gmail.com. Orcid iD: https://orcid.org/0000-0002-1724-3637

${ }^{4} \mathrm{E}-\mathrm{mail}$ : karolinealvesalm@gmail.com. Orcid iD: https://orcid.org/0000-0002-3078-5465

${ }^{5}$ E-mail: sobral.glebson@hotmail.com. Orcid iD: https://orcid.org/0000-0002-3835-7916

${ }^{6}$ E-mail: simonekameo@hotmail.com. Orcid iD https://orcid.org/0000-0002-0035-2415

7E-mail: glebsonmoura@yahoo.com.br. Orcid iD: https://orcid.org/0000-0002-4977-2787

Endereço para correspondência: Dayane Ketlyn da Cunha Santos. Avenida Governador Marcelo Déda, 13 - Jardim Campo Novo. Lagarto (SE), Brasil. CEP 49400-000. E-mail: daayketlyn27@gmail.com 


\section{INTRODUÇÃO}

O câncer é uma manifestação clínica caracterizada pela multiplicação desordenada de células em consequência de alteraçóes do seu ciclo de vida, dessa forma, o corpo perde a capacidade de controle do mecanismo de divisão celular e essas estruturas ganham a capacidade de se aderir e invadir outros tecidos orgânicos ${ }^{1}$. Para cada ano do triênio 2020-2022, estima-se que aproximadamente 625 mil novos casos ocorram, demonstrando a importância e a necessidade de discutir sobre o tema ${ }^{2}$.

No âmbito pediátrico, é notório que a neoplasia é cercada de medos e anseios que afetam a dinâmica do indivíduo, família e grupo social no qual este está inserido. Desse modo, a doença é acompanhada de estigmas que incentivam o surgimento de medidas de adaptação e enfrentamento desse problema ${ }^{3}$.

Estimativas epidemiológicas do câncer infantojuvenil são de grande relevância para o planejamento assistencial. Segundo o Instituto Nacional de Câncer José Alencar Gomes da Silva (INCA) ${ }^{2}$, estimam-se 4.310 casos novos para cada ano do triênio 2020-2022 em crianças e adolescentes, sendo mais frequente nas Regióes Sudeste (3.690), Nordeste (2.190) e Sul (1.310). Em contrapartida, as Regiōes Norte (630) e Centro-Oeste (640) apresentarão menos casos ${ }^{2}$.

A Política Nacional de Atenção Oncológica ${ }^{4}$ prevê a garantia de assistência diante de todas as instâncias de suporte oncológico, indo desde a prevençáo ao tratamento, respeitando a atençáo integral. Aliado a esse fator, a Lei $\mathrm{n}^{\circ}$. 12.732, de 22 de novembro de $2012^{5}$, estabelece que os pacientes oncológicos tenham acesso ao tratamento em um prazo de 60 dias após o diagnóstico da doença. Contudo, sabe-se que, na prática, o subfinanciamento do sistema de saúde, problemas associados à capacitação, fatores sociodemográficos e a falta de articulação em redes contribuem para o não cumprimento dessa regulamentaçãa $0^{5-7}$.

Desse modo, visando a intensificar e a verificar a aplicação desses pareceres legais, entra em vigor a Lei ${ }^{\circ}$. 13.685, de 25 de junho de $2018^{8}$, que obriga os serviços de assistência oncológica a notificarem os casos de câncer em todo o território, com a finalidade de verificar possíveis lacunas assistenciais ${ }^{8,6}$.

O monitoramento da rede de assistência oncológica é fundamental para a identificaçáo de entraves que podem prejudicar e, assim, atrasar o início do tratamento do paciente. Dessa forma, atualmente, em parceria com o INCA, o Ministério da Saúde integra o PAINEL-Oncologia ${ }^{9}$, que visa a fornecer informaçóes claras e detalhadas da realidade brasileira a respeito do intervalo entre o diagnóstico e o início do tratamento oncológico, permitindo ao usuário fazer análise dos dados por meio dos relatórios disponíveis e discutir o cenário encontrado. Trata-se de uma ferramenta ágil e acessível para o monitoramento do tempo do primeiro tratamento oncológico ${ }^{10}$.

Destarte, o presente estudo objetiva analisar os fatores associados à instituição do tratamento precoce nos casos de câncer infantojuvenil no Brasil entre os anos de 2013 e 2019, utilizando como banco de dados o PAINEL-Oncologia ${ }^{9}$, disponibilizado no Departamento de Informática do Sistema Único de Saúde (DATASUS).

\section{MÉTODO}

Trata-se de um estudo ecológico, com dados dos casos de todos os tipos de câncer diagnosticados no Brasil do ano de 2013 a 2019 na populaçáo infantojuvenil (0 a 19 anos) com o intuito de analisar os fatores associados à instituição do tratamento oncológico precoce.

As informações dos casos foram extraídas do PAINEL-Oncologia ${ }^{9}$ mediante acesso à base sem identificação em novembro de 2020, as quais também são oriundas do Sistema de Informaçáo Ambulatorial (SIA), por meio do Boletim de Produção Ambulatorial Individualizado (BPA-I) e da Autorização de Procedimento de Alta Complexidade (Apac), do Sistema de Informação Hospitalar (SIH) e do Sistema de Informaçôes de Câncer (Siscan), geridos pelo Ministério da Saúde, da Secretaria de Assistência à Saúde, em conjunto com as Secretarias Estaduais de Saúde e as Secretarias Municipais de Saúde, sendo processado pelo DATASUS, da Secretaria Executiva do Ministério da Saúde.

A definiçãoo de caso foi feita a partir da combinação do Cartão Nacional de Saúde (CNS) com a 10a Classificação Internacional de Doenças e Problemas Relacionados à Saúde (CID-10) ${ }^{11}$ informada. Sendo assim, um mesmo cartão com mais de uma CID representa casos diferentes na plataforma ${ }^{9,12}$.

Dessa maneira, foram selecionadas as seguintes variáveis:

a. Sexo: feminino; masculino.

b. Unidade Federativa (UF) do diagnóstico: permite selecionar os casos segundo o Estado do estabelecimento que realizou o diagnóstico, os quais foram agrupados por regióes geográficas e comparados com a realidade nacional.

c. Faixa etária: categorizada em lactentes e neonatos $(<2$ anos), pré-escolares ( 2 a 4 anos), escolares (5 a 10 anos), adolescentes (11 a 19 anos) e jovens ( 0 a 19 anos), como um grupo.

d. Diagnóstico: refere-se à neoplasia (CID-10) informada no exame de diagnóstico, agrupada em três categorias: "Neoplasias Malignas", que reúne os códigos de neoplasia maligna (C00-C97) excluindo as duas 
exceções que constam na Lei no. 12.732/125 (códigos C44 e C73); "Neoplasias in situ", que reúne os códigos D00-D09; e "Neoplasias de comportamento incerto ou desconhecido", que reúne os códigos D37-D48. Os códigos da CID-10 D00-D09 e D37-48 compuseram a categoria "Outras neoplasias".

e. Modalidade terapêutica: refere-se ao procedimento do primeiro tratamento, podendo ser cirurgia, quimioterapia, radioterapia e ambos (quimioterapia + radioterapia com a mesma data de tratamento). Dessa forma, a modalidade terapêutica foi agrupada como "cirúrgica" e "não cirúrgica".

f. Estadiamento: refere-se aos estadiamentos 0, I, II, III e IV registrados nos tratamentos de quimioterapia, radioterapia e em ambos. A cirurgia não possui informação de estadiamento, pois esse tratamento é recuperado do SIH, que não possui essa informação.

g. Tempo de tratamento: refere-se ao intervalo de tempo, em dias, calculado entre a data do exame diagnóstico e a data do primeiro tratamento; registrado em: 0 a 30 dias, 31 a 60 dias, mais de 60 dias e sem informação de tratamento. Posteriormente, foram dicotomizados em $\leq 30$ dias $>30$ dias. Assim, foi realizada a análise, considerando o intervalo de 30 dias antes do prazo máximo de 60 dias, estipulado pela referida lei. Os casos sem informação sobre o tempo de tratamento foram excluídos do estudo.

Os dados foram tabulados no Microsoft Excel $^{\circledR} 2016$. Posteriormente, foi realizada uma análise descritiva desses dados por meio da distribuição das variáveis da população de estudo. Por se tratar de variáveis categóricas, foram calculadas proporçóes, sendo a comparação entre os grupos realizada pelo teste do qui-quadrado de Pearson. Adicionalmente, foram estimadas as odds ratio (OR) pela regressão logística univariada, com seus respectivos intervalos de confiança de 95\% (IC95\%). Portanto, foi considerado o nível de significância de 5\% para os referidos testes estatísticos. Todas as análises foram realizadas com uso do software Statistical Package for Social Sciences (SPSS), versão 25.0. Ainda que a OR superestime a força de associação em estudos como este, comparada à razão de prevalências (RP), a escolha dessa medida de associação se deu pelo fato de a OR permitir a identificação de possíveis associaçóes nas quais a RP pode levar a falsas conclusôes $^{13,14}$.

Este estudo não foi submetido a um Comitê de Ética em Pesquisa por utilizar dados secundários.

\section{RESULTADOS}

Dos 49.723 casos de câncer infantojuvenil oriundos do PAINEL-Oncologia ${ }^{9}$ no período do estudo, não foram encontradas informaçóes sobre o tempo até o início do tratamento em 10.012 casos (20,13\%), o que levou à exclusão dessa parcela. Entre os 39.711 casos incluídos, 29.381 (74\%) realizaram o tratamento de forma precoce, isto é, 30 dias antes do prazo máximo estipulado pela Lei $\mathrm{n}^{\mathrm{o}} .12 .732 / 12^{5}$, e compuseram a casuística final analisada neste estudo.

Foram observadas menores chances de as neoplasias malignas terem tratamento precoce, comparadas a outras neoplasias. No entanto, náo foi observada diferença significativa conforme o sexo. Por outro lado, neoplasias que foram encaminhadas ao tratamento cirúrgico tiveram mais chances de o início do tratamento ocorrer em até 30 dias do diagnóstico, em relação às outras modalidades terapêuticas.

Referente ao total da população estudada, os adolescentes estavam mais propensos a um início do tratamento mais tardio, ao contrário do grupo de lactentes, neonatos e pré-escolares.

Além disso, houve diferença significativa no que diz respeito aos casos tratados em tempo oportuno entre as Regióes geopolíticas, sendo encontradas menores chances de haver tratamento precoce nas Regiōes Norte (OR 0, 399 [IC95\% 0,367-0,434]) e Sudeste (OR 0,920 [IC95\% 0,883-0,959]); e maiores chances nas Regiōes Sul (OR 1,430 [IC95\% 1,345-1,521]), Centro-Oeste (OR 1,316 [IC95\% 1,202-1,441]) e Nordeste (OR 1,065 [IC95\% 1,015-1,118]).

A Tabela 1 apresenta os dados das variáveis sociodemográficas, diagnósticas e terapêuticas de acordo com o tempo decorrido do diagnóstico da neoplasia até o início do seu tratamento.

Em relação ao total de casos, as neoplasias que apresentaram menores chances de terem seu tratamento iniciado em até 30 dias a partir do diagnóstico foram, em ordem crescente: C69-C72 (dos olhos, do encéfalo e de outras partes do Sistema Nervoso Central); C00-C14 (do lábio, cavidade oral e faringe); C51-C58 (dos órgãos genitais femininos); C45-C49 (do tecido mesotelial e tecidos moles); C43-C44 (melanoma e outras neoplasias malignas da pele); C76-C80 (de localizações mal definidas, secundárias e de localizações não especificadas); C60-C63 (dos órgãos genitais masculinos); e C40-C41 (dos ossos e das cartilagens articulares). A Tabela 2 apresenta o descritivo detalhado das neoplasias infantojuvenis de acordo com o tempo decorrido do diagnóstico até o início do tratamento.

Em contrapartida, as seguintes neoplasias tiveram maiores chances de ter tratamento precoce, em ordem decrescente: C15-C26 (dos órgãos digestivos); C50 (da mama); D37-D48 (de comportamento incerto ou desconhecido); C64-C68 (do trato urinário); C81-C96 (declaradas ou presumidas como primárias, dos tecidos 
Tabela 1. Dados das variáveis sociodemográficas, diagnósticas e terapêuticas, conforme o tempo decorrido do diagnóstico da neoplasia até o início do seu tratamento na população infantojuvenil brasileira, 2013-2019

\begin{tabular}{|c|c|c|c|c|c|}
\hline \multirow[b]{2}{*}{ Sexo } & \multicolumn{2}{|c|}{ Tempo até o tratamento } & \multirow[b]{2}{*}{ OR } & \multirow[b]{2}{*}{ IC (95\%) } & \multirow[b]{2}{*}{ p-value ${ }^{c}$} \\
\hline & Até 30 dias & $\begin{array}{l}\text { Mais de } 30 \\
\text { dias }\end{array}$ & & & \\
\hline Masculino & $\begin{array}{l}16.246 \\
(40,9 \%)\end{array}$ & $\begin{array}{l}5.795 \\
(14,6 \%)\end{array}$ & 0,968 & \multirow[t]{2}{*}{$0,925-1,013$} & \\
\hline Feminino & $\begin{array}{l}13.135 \\
(33,1 \%)\end{array}$ & $\begin{array}{c}4.535 \\
(11,4 \%)\end{array}$ & 1,0 & & 0,152 \\
\hline \multicolumn{6}{|l|}{ Diagnóstico } \\
\hline Neoplasias Malignas & $\begin{array}{l}27.737 \\
(79,3 \%)\end{array}$ & $\begin{array}{l}10.053 \\
(25,5 \%)\end{array}$ & 0,416 & $0,360-0,480$ & \multirow[b]{2}{*}{$<0,001$} \\
\hline $\begin{array}{l}\text { Outras neoplasias (in situ OU de } \\
\text { comportamento incerto) }\end{array}$ & $\begin{array}{c}1.434 \\
(3,7 \%)\end{array}$ & $\begin{array}{l}216 \\
(0,5 \%)\end{array}$ & 1,0 & & \\
\hline \multicolumn{6}{|l|}{ Modalidade terapêutica } \\
\hline Cirúrgica & $\begin{array}{l}8.390 \\
(21,1 \%)\end{array}$ & $\begin{array}{l}732 \\
(1,8 \%)\end{array}$ & 5,241 & $4,841-5,673$ & \multirow{2}{*}{$<0,001$} \\
\hline Não cirúrgica & $\begin{array}{l}20.991 \\
(52,9 \%)\end{array}$ & $\begin{array}{c}9.598 \\
(24,2 \%)\end{array}$ & 1,0 & & \\
\hline \multicolumn{6}{|l|}{ Faixa etária } \\
\hline $\begin{array}{l}\text { Lactentes e neonates } \\
\text { ( }<2 \text { anos) }\end{array}$ & $\begin{array}{l}3.139 \\
(7,9 \%)\end{array}$ & $\begin{array}{c}745 \\
(1,9 \%)\end{array}$ & 1,481 & $0,764-0,774$ & \multirow{4}{*}{$<0,001$} \\
\hline Pré-escolares (2 a 4 anos) & $\begin{array}{l}5.819 \\
(14,7 \%)\end{array}$ & $\begin{array}{c}1.424 \\
(3,6 \%)\end{array}$ & 1,437 & $1,350-1,529$ & \\
\hline Escolares (5 a 10 anos) & $\begin{array}{c}7.152 \\
(18,0 \%)\end{array}$ & $\begin{array}{l}2.319 \\
(5,8 \%)\end{array}$ & 1,084 & $1,029-1,142$ & \\
\hline $\begin{array}{l}\text { Adolescentes } \\
\text { (11 a } 19 \text { anos) }\end{array}$ & $\begin{array}{l}13.271 \\
(33,4 \%)\end{array}$ & $\begin{array}{l}5.842 \\
(14,7 \%)\end{array}$ & 0,799 & $0,769-0,830$ & \\
\hline Total (0-19 anos) & $\begin{array}{l}29.381 \\
(74,0 \%)\end{array}$ & $\begin{array}{l}10.330 \\
(26,0 \%)\end{array}$ & 1,0 & & \\
\hline \multicolumn{6}{|l|}{ Região } \\
\hline Norte & $\begin{array}{l}1.240 \\
(3,1 \%)\end{array}$ & $\begin{array}{l}1.092 \\
(2,2 \%)\end{array}$ & 0,399 & $0,367-0,434$ & \multirow{5}{*}{$\begin{array}{r}<0,001 \\
0,009 \\
0,020 \\
<0,001 \\
<0,001\end{array}$} \\
\hline Nordeste & $\begin{array}{l}8.366 \\
(21,1 \%)\end{array}$ & $\begin{array}{l}2.771 \\
(6,8 \%)\end{array}$ & 1,065 & $1,015-1,118$ & \\
\hline Sudeste & $\begin{array}{l}11.495 \\
(28,9 \%)\end{array}$ & $\begin{array}{c}4.391 \\
(11,0 \%)\end{array}$ & 0,920 & $0,883-0,959$ & \\
\hline Sul & $\begin{array}{l}5.907 \\
(14,8 \%)\end{array}$ & $\begin{array}{c}1.452 \\
(3,5 \%)\end{array}$ & 1,430 & $1,345-1521$ & \\
\hline Centro-Oeste & $\begin{array}{l}2.373 \\
(6,0 \%)\end{array}$ & $\begin{array}{c}634 \\
(1,6 \%) \\
\end{array}$ & 1,316 & $1,202-1,441$ & \\
\hline Brasil & $\begin{array}{l}2.9381 \\
(74,0 \%)\end{array}$ & $\begin{array}{l}10.330 \\
(26,0 \%)\end{array}$ & 1,0 & & \\
\hline
\end{tabular}

(a) Teste do qui-quadrado de Pearson $\left(\chi^{2}\right)$.

linfático, hematopoético e tecidos correlatos); e C73-C75 (da tireoide e de outras glândulas endócrinas). As neoplasias com CID-10 C30-C39 (do aparelho respiratório e dos órgãos intratorácicos) não tiveram risco significativamente diferente em relação ao total estudado, e todas as neoplasias in situ foram tratadas precocemente.

\section{DISCUSSÃO}

O tratamento oportuno é essencial para um prognóstico favorável do câncer na infância e na adolescência, impactando diretamente na morbimortalidade dessa faixa etária. $\mathrm{O}$ atraso na terapêutica pode decorrer de diversos fatores, como diagnóstico incorreto, lista 
Tabela 2. Neoplasias infantojuvenis de acordo com o tempo decorrido do diagnóstico até o início do seu tratamento, Brasil, 2013-2019

\begin{tabular}{|c|c|c|c|c|c|}
\hline \multirow[b]{2}{*}{ Neoplasias malignas } & \multicolumn{2}{|c|}{ Tempo até o tratamento } & \multirow[b]{2}{*}{ OR } & \multirow[b]{2}{*}{ IC (95\%) } & \multirow[b]{2}{*}{ p-value ${ }^{a}$} \\
\hline & Até 30 dias & $\begin{array}{l}\text { Mais de } 30 \\
\text { dias }\end{array}$ & & & \\
\hline $\begin{array}{l}\text { (COO-C14) Neoplasia [tumores] } \\
\text { malignas, lábio, cavidade oral e } \\
\text { faringe }\end{array}$ & $\begin{array}{c}460 \\
(1,16 \%)\end{array}$ & $\begin{array}{c}301 \\
(0,76 \%)\end{array}$ & 0,537 & $0,464-0,622$ & $<0,05$ \\
\hline $\begin{array}{l}\text { (C15-C26) Neoplasia malignas, } \\
\text { órgãos digestivos }\end{array}$ & $\begin{array}{l}1.130 \\
(2,85 \%)\end{array}$ & $\begin{array}{c}165 \\
(0,42 \%)\end{array}$ & 2,408 & $2,042-2,839$ & \\
\hline $\begin{array}{l}\text { (C40-C41) Neoplasias [tumores] } \\
\text { malignas(os) dos ossos e das } \\
\text { cartilagens articulares }\end{array}$ & $\begin{array}{l}2.164 \\
(5,45 \%)\end{array}$ & $\begin{array}{c}867 \\
(2,18 \%)\end{array}$ & 0,878 & $0,809-0,952$ & \\
\hline $\begin{array}{l}\text { (C43-C44) Melanoma e outras(os) } \\
\text { neoplasias [tumores] malignas(os) } \\
\text { da pele }\end{array}$ & $\begin{array}{c}177 \\
(0,45 \%)\end{array}$ & $\begin{array}{c}101 \\
(0,25 \%)\end{array}$ & 0,616 & $0,482-0,788$ & \\
\hline $\begin{array}{l}\text { (C45-C49) Neoplasias [tumores] } \\
\text { malignas(os) do tecido mesotelial e } \\
\text { tecidos moles }\end{array}$ & $\begin{array}{c}1.862 \\
(4,69 \%)\end{array}$ & $\begin{array}{c}1099 \\
(2,77 \%)\end{array}$ & 0,596 & $0,551-0,644$ & \\
\hline $\begin{array}{l}\text { (C50) Neoplasias [tumores] } \\
\text { malignas(os) da mama }\end{array}$ & $\begin{array}{c}257 \\
(0,65 \%)\end{array}$ & $\begin{array}{c}40 \\
(0,10 \%)\end{array}$ & 2,259 & $1,618-3,154$ & \\
\hline $\begin{array}{l}\text { (C51-C58) Neoplasias [tumores] } \\
\text { malignas(os) dos órgãos genitais } \\
\text { femininos }\end{array}$ & $\begin{array}{c}649 \\
(1,63 \%)\end{array}$ & $\begin{array}{c}402 \\
(1,01 \%)\end{array}$ & 0,568 & $0,500-0,644$ & \\
\hline $\begin{array}{l}\text { (C60-C63) Neoplasias [tumores] } \\
\text { malignas(os) dos órgãos genitais } \\
\text { masculinos }\end{array}$ & $\begin{array}{l}539 \\
(1,36 \%)\end{array}$ & $\begin{array}{c}225 \\
(0,57 \%)\end{array}$ & 0,842 & $0,720-0,989$ & \\
\hline $\begin{array}{l}\text { (C64-C68) Neoplasias [tumores] } \\
\text { malignas(os) do trato urinário }\end{array}$ & $\begin{array}{c}1.539 \\
(3,88 \%)\end{array}$ & $\begin{array}{c}293 \\
(0,74 \%)\end{array}$ & 1,847 & $1,627-2,097$ & \\
\hline $\begin{array}{l}\text { (C69-C72) Neoplasias [tumores] } \\
\text { malignas(os) dos olhos, do encéfalo } \\
\text { e de outras partes do Sistema } \\
\text { Nervoso Central }\end{array}$ & $\begin{array}{l}2.755 \\
(6,94 \%)\end{array}$ & $\begin{array}{l}2.247 \\
(5,66 \%)\end{array}$ & 0,431 & $0,406-0,458$ & \\
\hline $\begin{array}{l}\text { (C73-C75) Neoplasias [tumores] } \\
\text { malignas(os) da tireoide e de outras } \\
\text { glândulas endócrinas }\end{array}$ & $\begin{array}{c}844 \\
(2,13 \%)\end{array}$ & $\begin{array}{c}255 \\
(0,64 \%)\end{array}$ & 1,164 & $1,010-1,341$ & \\
\hline $\begin{array}{l}\text { (C76-C80) Neoplasias [tumores] } \\
\text { malignas(os) de localizações } \\
\text { mal definidas, secundárias e de } \\
\text { localizações não especificadas }\end{array}$ & $\begin{array}{c}1.257 \\
(3,17 \%)\end{array}$ & $\begin{array}{c}565 \\
(1,42 \%)\end{array}$ & 0,782 & $0,707-0,866$ & \\
\hline $\begin{array}{l}\text { (C81-C96) Neoplasias [tumores] } \\
\text { malignas(os), declaradas ou } \\
\text { presumidas como primárias, dos } \\
\text { tecidos linfático, hematopoético e } \\
\text { tecidos correlatos }\end{array}$ & $\begin{array}{c}13.914 \\
(35,04 \%)\end{array}$ & $\begin{array}{l}3.401 \\
(8,56 \%)\end{array}$ & 1,438 & $1,377-1,503$ & \\
\hline $\begin{array}{l}\text { (D00-D09) Neoplasias [tumores] in } \\
\text { situ }\end{array}$ & $\begin{array}{c}108 \\
(0,27 \%)\end{array}$ & $\begin{array}{c}0 \\
(0 \%)\end{array}$ & - & & \\
\hline $\begin{array}{l}\text { (D37-D48) Neoplasias [tumores] } \\
\text { de comportamento incerto ou } \\
\text { desconhecido }\end{array}$ & $\begin{array}{l}1.326 \\
(3,34 \%)\end{array}$ & $\begin{array}{c}216 \\
(0,54 \%)\end{array}$ & 2,158 & $1,866-2,497$ & \\
\hline \multirow[t]{2}{*}{$\begin{array}{l}\text { (C30-C39) Neoplasias [tumores] } \\
\text { malignas(os) do aparelho } \\
\text { respiratório e dos órgãos } \\
\text { intratorácicos }\end{array}$} & $\begin{array}{c}400 \\
(1,01 \%)\end{array}$ & $\begin{array}{c}153 \\
(0,39 \%)\end{array}$ & 0,919 & $0,762-1,109^{b}$ & $1,109^{b}$ \\
\hline & $\begin{array}{l}29.381 \\
(74,0 \%)\end{array}$ & $\begin{array}{l}10.330 \\
(26,0 \%)\end{array}$ & 1,0 & & \\
\hline
\end{tabular}

(a) Teste do qui-quadrado de Pearson $\left(\chi^{2}\right)$.

(b) Resultado não significativo $(\mathrm{p}=0,198)$ pelo teste de Pearson. 
de espera e descentralização do cuidado, o que leva a encaminhamentos e deslocamentos intermunicipais ou interestaduais ${ }^{15}$.

De acordo com a habilitação na atenção especializada em Oncologia, conforme a Portaria MS/SAES no 1.399, de 17 de dezembro de $2019^{16}$, os estabelecimentos de tratamento foram divididos. Assim, o diagnóstico, estadiamento e tratamento oncológico podem ser realizados nos seguintes locais: Unidades de Assistência de Alta Complexidade em Oncologia (Unacon), quando possuem condiçôes técnicas e recursos humanos para prestar assistência no diagnóstico e tratamento dos cânceres mais prevalentes; Centros de Assistência de Alta Complexidade em Oncologia (Cacon), os quais prestam assistência para todos os tipos de neoplasias; Centros de Referência de Alta Complexidade em Oncologia, que são Cacon com alguns atributos, a exemplo de ser um Hospital de Ensino e estar presente em um território a depender da quantidade de casos novos de câncer na população; e Hospitais Gerais com Cirurgia Oncológica ${ }^{4}$. É importante destacar, ainda, que o PAINEL-Oncologia', de onde foram extraídos os dados do presente estudo, traz informaçóes de casos diagnosticados e tratados também fora das unidades habilitadas.

Os adolescentes têm o início do tratamento mais tardio quando comparados a grupos de menor faixa etária, como lactentes, neonatos e pré-escolares. Tal achado pode estar associado ao nível de monitorização pelos cuidadores e pela maior frequência de consultas, características de crianças de menor idade ${ }^{17}$. Além disso, os adolescentes já apresentam mais discernimento e compreensão dos impactos físicos e psicoemocionais da doença e do seu tratamento, podendo mostrar-se resistentes ao seu início e continuaçãa ${ }^{18}$. Essa perspectiva foi vista, também, no estudo Handayani et al. ${ }^{19}$ quando os maiores atrasos identificados foram em pacientes com idade maior ou igual a 10 anos.

Ademais, outro fator de grande relevância é o tipo do tumor, que impacta na resolutividade, morbimortalidade e qualidade de vida do paciente. Também foi observada essa correlação entre o tipo de câncer e o tempo para início do tratamento no estudo de Njuguna et al. ${ }^{20}$ realizado com pacientes oncológicos pediátricos no Quênia, no qual o atraso do tratamento foi mais longo para o sarcoma de Kaposi e menos longo para linfoma não Hodgkin e leucemia mieloide aguda.

O presente estudo revelou que as neoplasias dos tecidos linfáticos, hematopoéticos e correlatos apresentaram maiores chances de tratamento precoce. Entretanto, entre as que apresentaram menores chances de terem seu tratamento iniciado, em até 30 dias a partir do diagnóstico, destacam-se as neoplasias de Sistema Nervoso
Central e dos olhos. Desse modo, partindo do ponto de vista epidemiológico, é importante destacar que, nessa população, a leucemia aguda é a principal neoplasia na população pediátrica, seguida dos tumores do Sistema Nervoso Central ${ }^{21}$. Pontua-se, ainda, que o retinoblastoma é o tumor maligno ocular mais frequente na infância ${ }^{21}$.

$\mathrm{Na}$ análise dos dados, foi observado que alguns tumores tiveram seu tratamento iniciado mais tardiamente; ou seja, com mais de 30 dias, a exemplo dos tumores do Sistema Nervoso Central. Possivelmente esse atraso no tratamento pode ser decorrente da dificuldade de acesso ao sistema de saúde especializado, uma vez que, em razão da complexidade dos cânceres na faixa etária estudada, o tratamento deve ser feito em centro especializado ${ }^{21}$. O mesmo padrão foi descrito no Panorama da Oncologia Pediátrica do Rio de Janeiro ${ }^{22}$, em 2019, quando o início do tratamento dos tumores do Sistema Nervoso Central aconteceu em até 46 dias após o diagnóstico estabelecido. Além disso, um fator importante que pode explicar a necessidade de mais tempo até a chegada à instituição terapêutica é a transferência até o local de tratamento, pois, a depender do tipo de câncer, nem todas as Unacon ofertam o serviço, o que pode levar a um atraso de uma semana ou mais, como evidenciado no estudo de Mou et al. ${ }^{15}$.

Em contrapartida, notou-se que alguns tipos de tumores tiveram tratamento precoce como, por exemplo, tumores primários (tecidos linfáticos, hematopoéticos e tecidos correlatos). Esse padrão também foi encontrado no estudo de Handayani et al. ${ }^{19}$ realizado na Indonésia, cujos tumores neurológicos tiveram mais atrasos e os hematológicos, tempo mais curto para o início do tratamento.

Observou-se, ainda, que as neoplasias malignas apresentam maiores chances de tratamento tardio. Esse atraso no tratamento do câncer infantojuvenil é um importante fator para piores prognósticos, pois, de modo geral, o câncer pediátrico é mais agressivo e progressivo quando comparado com os cânceres em adultos ${ }^{23}$. Nesse sentido, o tratamento precoce se torna fundamental para melhores desfechos. Corroborando tais achados, Hanna et al. ${ }^{24}$ verificaram que o atraso no tratamento de quatro semanas está associado a um aumento no risco de morte que varia de $6 \%$ a $13 \%$ de acordo com o tipo de tumor e de tratamento ${ }^{24}$.

Além disso, é de suma importância entender o impacto da necessidade de transferência do paciente para a realização do tratamento após o diagnóstico, uma vez que essa necessidade esteve significativamente correlacionada com a maior extensão do intervalo diagnóstico-tratamento ${ }^{15}$. Assim, é imperioso compreender a diferença encontrada no tempo de tratamento a depender da modalidade terapêutica e da regiáo geográfica brasileira. 
O tratamento do câncer pretende curar, prolongar a vida e melhorar a qualidade de vida. Para tanto, conta, geralmente, com três formas de tratamento: cirurgia, radioterapia e quimioterapia ${ }^{1}$. O presente estudo revelou que as neoplasias encaminhadas ao tratamento cirúrgico tiveram maiores chances de início do tratamento em até 30 dias a partir do diagnóstico em detrimento das outras modalidades, o que pode ser explicado pelo fato de a distribuição das internaçóes hospitalares do SUS - que possibilitam procedimentos clínicos e cirúrgicos estarem mais difundidas no país do que a distribuição de radioterapia e quimioterapia, que geralmente são realizadas em hospitais credenciados com alta complexidade ${ }^{25}$.

Quanto às diferenças no tempo para a instituição do tratamento entre as Regióes brasileiras, os resultados apontaram menores chances de iniciar a terapêutica rapidamente na Região Norte, o que possivelmente está associado às políticas públicas e à distribuição dos serviços. $\mathrm{O}$ estudo de Grabois et al..$^{25}$, que objetivou analisar os fluxos de viagens de crianças e adolescentes com câncer, entre os locais de residência e serviço de saúde atendidos no SUS, evidenciou que os residentes dessa região precisaram percorrer maiores distâncias para receber o tratamento, já que a maioria das redes estava polarizada em torno das capitais dos Estados. Ademais, outro estudo apontou que alguns casos advindos de cidades localizadas nos Estados do Pará e Maranhão precisavam percorrer mais de mil quilômetros até a unidade de oncologia do hospital público pesquisado ${ }^{26}$.

Além disso, Grabois et al..$^{27}$ também destacaram, em outra pesquisa, que moradores das Regiáo Norte tiveram menos acesso à quimioterapia, à radioterapia, à internação e à cirurgia oncológica pediátrica. $\mathrm{O}$ mesmo foi visto para a Região Nordeste, sobretudo no que diz respeito às regiôes de saúde mais periféricas. Entretanto, este estudo apontou essa Regiáo com maiores chances de o tratamento ser instituído em até 30 dias do que, por exemplo, a Região Sudeste. Nesse contexto, as maiores chances de instituir o tratamento em até 30 dias foram encontradas nas Regiōes Sul, Centro-Oeste e Nordeste.

Assim como há uma variação regional no tempo para iniciar o tratamento, existem também variaçôes referentes à mortalidade, uma vez que esta depende das condiçóes de acesso, uso e desempenho dos serviços de saúde, fatores de risco neoplásicos, qualidade dos dados do Sistema de Informação sobre Mortalidade (SIM) ${ }^{28}$, pois há altas proporções de causas mal definidas de morte no Norte e Nordeste ${ }^{29}$. Isso também pode estar associado aos resultados referentes à instituição terapêutica. Assim, torna-se nítida a importância da distribuição dos serviços visando a atender às demandas da populaçáo de determinada Regiáo de modo a promover uma maior equidade entre os Estados ${ }^{25}$.
Por fim, outro ponto que merece destaque é a falta de informaçôes detalhadas sobre o tratamento de 10.012 casos $(20,13 \%)$. Nesse sentido, Jorge et al. ${ }^{30}$, em extenso estudo sobre a qualidade das informaçóes lançadas nos diversos Sistemas de Informação em Saúde do SUS, encontraram desfalques importantes tanto no que tange à completitude dos casos notificados em relaçáo ao universo - definido com a ajuda de outras bases de dados - como à qualidade dos dados em si, de acordo com a frequência dos campos de preenchimento "ignorados". Portanto, embora não se trate de uma limitação específica deste estudo, mas de todos aqueles que utilizam informaçóes secundárias a bases correlatas ao PAINEL-Oncologia ${ }^{9}$, a minoração do detalhamento dos casos de câncer notificados empobrece as análises da qualidade assistencial prestada ${ }^{30}$.

Entre as limitaçôes deste estudo, destaca-se que apenas os casos diagnosticados e com informação de tratamento disponibilizados na plataforma PAINEL-Oncologia ${ }^{9}$ foram analisados, o que afeta diretamente a análise e a interpretação dos dados. Além disso, a literatura carece de estudos sobre a temática, o que dificulta a comparação dos resultados encontrados. Ademais, a escolha da OR como medida de associação pode ter superestimado a força das associaçóes encontradas. Diante disso, verifica-se a necessidade de novos estudos, a fim de promover um maior conhecimento a respeito do tempo para início do tratamento oncológico na população infantojuvenil.

\section{CONCLUSÃO}

A análise dos fatores associados à instituição do tratamento oportuno nos casos de câncer infantojuvenil no Brasil entre 2013 e 2019 mostrou que não há grande necessidade de redução no tempo, entretanto é crucial para um melhor desfecho nos casos de cânceres infantojuvenis.

Os achados deste estudo apontam dados essenciais, em consonância com a literatura, para visualização da situação atual do tratamento oncológico na população infantojuvenil no Brasil, ao mesmo tempo que demostram problemáticas que, se solucionadas, podem contribuir significativamente para a queda da morbimortalidade.

\section{CONTRIBUIÇÕES}

Todos os autores contribuíram substancialmente na concepção e/ou planejamento do estudo; na obtenção, análise e/ou interpretação dos dados; na redação e revisão crítica; e aprovaram a versão final a ser publicada.

\section{DECLARAÇÃO DE CONFLITO DE INTERESSES}

Nada a declarar. 


\section{FONTES DE FINANCIAMENTO}

Não há.

\section{REFERÊNCIAS}

1. Instituto Nacional de Câncer José Alencar Gomes da Silva. ABC do câncer: abordagens básicas para o controle do câncer [Internet]. 6 ed. rev. atual. Rio de Janeiro: INCA; 2020. [acesso 2021 fev 20]. Disponível em: https://www.inca.gov.br/sites/ufu.sti.inca.local/files// media/document//livro-abc-6-edicao-2020.pdf

2. Instituto Nacional de Câncer José Alencar Gomes da Silva. Estimativa 2020: incidência de câncer no Brasil [Internet]. Rio de Janeiro: INCA; 2019 [acesso 2021 jan 19]. Disponível em: https://www.inca.gov.br/sites/ ufu.sti.inca.local/files/media/document/estimativa-2020incidencia-de-cancer-no-brasil.pdf

3. Caprini FR, Motta AB. Câncer infantil: uma análise do impacto do diagnóstico. Psicol Teor Prát. 2017;19(2):16476. doi: http://doi.org/10.5935/1980-6906/psicologia. v19n2p161-173

4. Conselho Nacional de Secretários de Saúde (BR). Política Nacional de Atenção Oncológica [Internet]. Brasília, DF: Ministério da Saúde; 2005 [acesso 2021 jan 9]. Disponível em: http://bvsms.saude.gov.br/bvs/ publicacoes/politica_nacional_atencao_oncologica.pdf

5. Ministério da Saúde (BR). Lei $\mathrm{n}^{\circ} 12.732$, de 22 de novembro de 2012. Dispóe sobre o primeiro tratamento de paciente com neoplasia maligna comprovada e estabelece prazo para seu início [Internet]. Diário Oficial da União. 2012 nov 23 [acesso 2021 jan 10]; Seção 1:1. Disponível em: https://www2.camara.leg.br/legin/ fed/lei/2012/lei-12732-22-novembro-2012-774660publicacaooriginal-138184-pl.html

6. Lombardo MS, Popim RC. Acesso do paciente à rede oncológica na vigência da "Lei dos Sessenta Dias": revisão integrativa. Rev Bras Enferm. 2020;73(5):e20190406. doi: https://doi.org/10.1590/0034-7167-2019-0406

7. ValleTD, Turrini RNT, Poveda VB. Fatores intervenientes para o início do tratamento de pacientes com câncer de estômago e colorretal. Rev Latino-Am Enfermagem. 2017;25:e2879. doi: https://doi.org/10.1590/15188345.1493.2879

8. Presidência da República (BR). Lei n ${ }^{\circ} 13.685$, de 25 de junho de 2018. Altera a Lei n ${ }^{\circ} 12.732$, de 22 de novembro de 2012, para estabelecer a notificação compulsória de agravos e eventos em saúde relacionados às neoplasias, $\mathrm{e}$ a Lei $n^{\circ} 12.662$, de 5 de junho de 2012, para estabelecer a notificação compulsória de malformaçôes congênitas [Internet]. Diário Oficial da Uniáo. 2018 jun 26 [acesso 2021 jan 15]; Seção 1:1. Disponível em: http://www. planalto.gov.br/ccivil_03/_Ato2015-2018/2018/Lei/ L13685.htm
9. PAINEL-Oncologia [Internet]. Brasília, DF: DATASUS. [2019] - [atualização 2021 nov 15; acesso 2021 jan 22]. Disponível em: http://tabnet. datasus.gov.br/cgi/dhdat.exe?PAINEL_ONCO/ PAINEL_ONCOLOGIABR.def

10. Atty ATM, Jardim BC, Dias MBK, et al. PAINELOncologia: uma ferramenta de gestáo. Rev Bras Cancerol. 2020;66(2):e-04827. doi: https://doi. org/10.32635/2176-9745.RBC.2020v66n2.827

11. Organização Mundial da Saúde. CID-10: classificação estatística internacional de doenças e problemas relacionados à saúde. 10. ed. São Paulo: Edusp; 2017.

12. Antunes JLF, Cardoso MRA. Uso da análise de séries temporais em estudos epidemiológicos. Epidemiol Serv Saúde. 2015;24(3):565-76. doi: https://doi.org/10.5123/ S1679-49742015000300024

13. Thompson ML, Myers JE, Kriebel D. Prevalence odds ratio or prevalence ratio in the analysis of cross sectional data: what is to be done? Occup Environ Med. 1998;55(4):272-7. doi: https://doi.org/10.1136/ oem.55.4.272

14. Pearce N. Effect measures in prevalence studies. Environ Health Prospect. 2004;112(10):1047-50. doi: https:// doi.org/10.1289/ehp.6927

15. Mou J, Bolieu EL, Pflugeisen BM, et al. Delay in treatment after cancer diagnosis in adolescents and young adults: does facility transfer matter? J Adolesc Young Adult Oncol. 2019;8(3):243-53. doi: https://doi. org/10.1089/jayao.2018.0128

16. Ministério da Saúde (BR), Secretaria de Atenção Especializada. Portaria no 1.399 , de 17 de dezembro de 2019. Redefine os critérios e parâmetros referenciais para a habilitação de estabelecimentos de saúde na alta complexidade em oncologia no âmbito do SUS [Internet]. Diário Oficial da União. 2019 dez 19 [acesso 2021 maio 26]; Seção 1:173. Disponível em: https:// www.inca.gov.br/sites/ufu.sti.inca.local/files//media/ document//portaria_1399_17dez2019.pdf

17. Dang-Tan T, Trottier H, Mery LS, et al. Determinants of delays in treatment initiation in children and adolescents diagnosed with leukemia or lymphoma in Canada. Int J Cancer. 2010;126(8):1936-43. doi: https://doi. org/10.1002/ijc. 24906

18. Siqueira HBOM, Pelegrin AKAP, Gomez RRF, et al. Percepção de adolescentes com câncer: pesquisa fenomenológica. Rev Abordagem Gestalt [Internet]. 2015 [acesso 2021 jan 10];21(1):13-21. Disponível em: http://pepsic.bvsalud.org/scielo.php?script $=$ sci arttext\&pid=S1809-68672015000100003\&lng=pt \& $\mathrm{nrm}=$ iso

19. Handayani K, Sitaresmi MN, Supriyadi E, et al. Delays in diagnosis and treatment of childhood cancer in Indonesia. Pediatric Blood Cancer [Internet]. 2016;63(12):2189-96. doi: https://doi.org/10.1002/pbc.26174 
20. Njuguna F, Martijn $\mathrm{H}$, Langat $S$, et al. Factors influencing time to diagnosis and treatment among pediatric oncology patients in Kenya. Pediatric Hematol Oncol. 2016;33(3):186-99. doi: https://doi.org/10.3109/0888 0018.2016.1169566

21. Ministério da Saúde (BR), Secretaria de Atenção à Saúde, Departamento de Atenção Especializada e Temática. Protocolo de diagnóstico precoce do câncer pediátrico [Internet]. Brasília, DF: Ministério da Saúde; 2017 [acesso 2021 maio 21]. Disponível em: http://bvsms. saude.gov.br/bvs/publicacoes/protocolo_diagnostico_ precoce_cancer_pediatrico.pdf

22. Panorama da Oncologia Pediátrica [Internet]. Rio de Janeiro: Instituto Desiderata. Vol. 6, No. 6, nov 2019 - [acesso 2021 jan 15]. Disponível em: https:// desiderata.org.br/wp/wp-content/uploads/2019/11/ Boletim_PANORAMA-ONCOLOGIA_2019_WEB_ completo.pdf

23. Hora SS, Monteiro MVC, Dias SM, et al. Acesso e adesão ao tratamento oncológico infantojuvenil: para além do aspecto médico-biológico. Rev Bras Cancerol. 2018;64(3):405-8. doi: https://doi.org/10.32635/21769745.RBC.2018v64n3.48

24. Hanna TP, King WD, Thibodeau S, et al. Mortality due to cancer treatment delay: systematic review and meta-analysis. BMJ. 2020;371:m4087. doi: https://doi. org/10.1136/bmj.m4087

25. Grabois MF, Oliveira EXG, Carvalho MS. Assistência ao câncer entre crianças e adolescentes: mapeamento dos fluxos origem-destino no Brasil. Rev Saúde Públ. 2013;47(2):368-78. doi: https://doi.org/10.1590/ S0034-8910.2013047004305/

26. Mutti CF, Cruz VG, Santos LF, et al. Perfil clínico-epidemiológico de crianças e adolescentes com câncer em um serviço de oncologia. Rev Bras Cancerol. 2018;64(3):293-300. doi: https://doi. org/10.32635/2176-9745.RBC.2018v64n3.26

27. Grabois MF, Oliveira EXG, Carvalho MS. Childhood cancer and pediatric oncologic care in Brazil: access and equity. Cad Saúde Pública. 2011;27(9):1711-20. doi: https://doi.org/10.1590/S0102-311X2011000900005

28. SIM: Sistema de Informação sobre Mortalidade [Internet]. Brasília (DF): DATASUS. c2008 - [acesso 2021 maio 21]. Disponível em: http://tabnet.datasus. gov.br/cgi/tabcgi.exe?sim/cnv/obt10uf.def

29. Instituto Nacional de Câncer. A situação do câncer no Brasil [Internet]. Rio de Janeiro: INCA; 2006 [acesso 2021 fev 21]. Disponível em: https://bvsms.saude.gov. br/bvs/publicacoes/situacao_cancer_brasil.pdf
30. Jorge MHPM, Laurenti R, Gotlieb SLD. Avaliação dos sistemas de informação em saúde no Brasil. Cad Saúde Colet [Internet]. 2010 [acesso 2021 jan 10];18(1):0718. Disponível em: http://www.cadernos.iesc.ufrj. $\mathrm{br} /$ cadernos/images/csc/2010_1/artigos/Modelo\%20 Livro\%20UFRJ\%201-a.pdf 